\title{
Blast Domination for Mycielski's Graph of Graphs
}

\author{
K. Ameenal Bibi, P.Rajakumari
}

\begin{abstract}
The hub of this article is a search on the behavior of the Blast domination and Blast distance-2 domination for Mycielski's graph of some particular graphs and zero divisor graphs.
\end{abstract}

Key Words:Blast domination number, Blast distance-2 domination number, Mycielski'sgraph.

\section{INTRODUCTION}

The concept of triple connected graphs was introduced by Paulraj Joseph et.al [9]. A graph is said to be triple connected if any three vertices lie on a path in G. In [6] the authors introduced triple connected domination number of a graph. A subset $\mathrm{D}$ of $\mathrm{V}$ of a nontrivial graph $\mathrm{G}$ is said to betriple connected dominating set, if $\mathrm{D}$ is a dominating set and $\langle\mathrm{D}\rangle$ is triple connected. The minimum cardinality taken over all triple connected dominating sets is called the triple connected domination number of $\mathrm{G}$ and is denoted by $\gamma_{t c}(G)$. Also Mahadevan et.al introduced the concept of complementary triple connected domination number of a graph and A.Ahilaet.al.,introducedBlast Domination Number of a graph with a real life application.

\section{THE MYCIELSKI CONSTRUCTION [5]}

The open neighborhood of a vertex $v$ in a graph $\mathrm{G}$ denoted by $N_{G}(v)$ isthe set of all vertices of $G$, which are adjacent to $v$. Also, $N_{G}[v]=N_{G}(v) \cup\{v\}$ is called the closed neighborhood of $v$ in the graph $\mathrm{G}$. In this paper, by $\mathrm{G}$ one means a connected graph.

From a graph $\mathrm{G}$, by Mycielski's construction one can get a graph $\mu$ (G) with $V(\mu(G))=V \cup U \cup\{w\}$, where $V=V(G)=\left\{v_{1}, \ldots v_{n}\right\}, U=\left\{u_{1}, \ldots, u_{n}\right\}$, and $E(\mu(G))=E(G) \cup\left\{u_{i} v: v \in N_{G}\left(v_{i}\right) \cup\{w\}, i=\right.$ $1, \ldots, n\}$.For each $0 \leq i \leq n, v_{i}$ and $u_{i}$ are called the corresponding vertices of $\mu(\mathrm{G})$ and denote $C\left(v_{i}\right)=$ $u_{i}, C\left(u_{i}\right)=v_{i}$. Moreover, for subsets $\mathrm{A} \subseteq \mathrm{U}, \mathrm{B} \subseteq \mathrm{V}$, one denotes: $C(A)=\left\{C\left(u_{i}\right): u_{i} \in A\right\}, C(B)=\left\{C\left(v_{i}\right):\right.$ $\left.v_{i} \in B\right\}$. Also, $\mathrm{x} \leftrightarrow \mathrm{y}$ is denoted, when $\{\mathrm{x}, \mathrm{y}\}$ is an edge.

\section{Definition 2.1[7]}

A graph $G$ is said to be triple connected, if any three vertices of $G$ lie on a path.

\section{Definition 2.2[10]}

A set $D$ of vertices in a graph $G$ is a distance- 2 dominating set if every vertex in $V-D$ is within distance-2 of atleast one vertex in $D$. The distance-2 domination number $\gamma_{\leq 2}(G)$

Revised Manuscript Received on 14 August, 2019.

K. AmeenalBibi, P.G. and Research Department of Mathematics, D.K.M.College for Women (Autonomous), Vellore, Tamilnadu, India.

P.Rajakumari, P.G. and Research Department of Mathematics, D.K.M.College for Women (Autonomous), Vellore, Tamilnadu, India. (Email: rajakumari0990@gmail.com). is the minimum cardinality of a distance-2 dominating set in G.

\section{Definition 2.3[7]}

A non-empty subset $D$ of $V$ of a connected graph $G$ is called a Blast dominating set, if $D$ is aconnected dominating set and the induced sub graph $\langle V-D\rangle$ is triple connected. The minimum cardinality taken over all such Blast dominating sets is called the Blast domination number of $G$ and is denoted by $\gamma_{c}^{t c}(G)$.

\section{Definition2.4}

A non-empty subset $D$ of vertices in a graph $G$ is a blast distance-2 dominating set if every vertex in $V-D$ is within distance-2 of atleast one vertex in $D$. The blast distance-2 domination number $\gamma_{c \leq 2}^{t c}(G)$ is the minimum cardinality of a blast distance-2 dominating set in $G$.

\section{Definition 2.5}

A distance -2dominating set $\mathrm{D} \subseteq \mathrm{V}$ of a graph $\mathrm{G}$ is an independentdistance -2dominating set if the induced sub graph <D> has no edges. The independent distance 2 domination number $i_{\leq 2}(G)$ is the minimum cardinality of a minimal independentdistance -2dominating set.

The minimal independent distance -2 dominating set in a graph $\mathrm{G}$ is an independent distance -2 dominating set that contains no independent distance - 2 dominating set as a proper subset.

The distance -2 open neighborhood of a vertex $v \in V$ is the set, $N_{\leq 2}(v)$ of vertices within distance of two from $v$.

\section{MAIN RESULTS}

Blast domination in the Mycielski's graph of $C_{n}, K_{n}$ , $W_{n} K_{m, n}, F_{1, n}, T_{m, n}$, and $T_{n}$ graphs

In this section, blast domination number of some graphs are investigated. Many bounds for this parameter is obtained.

Example 3.1 


\section{BLAST DOMINATION FOR MYCIELSKI'S GRAPH OFGRAPHS}

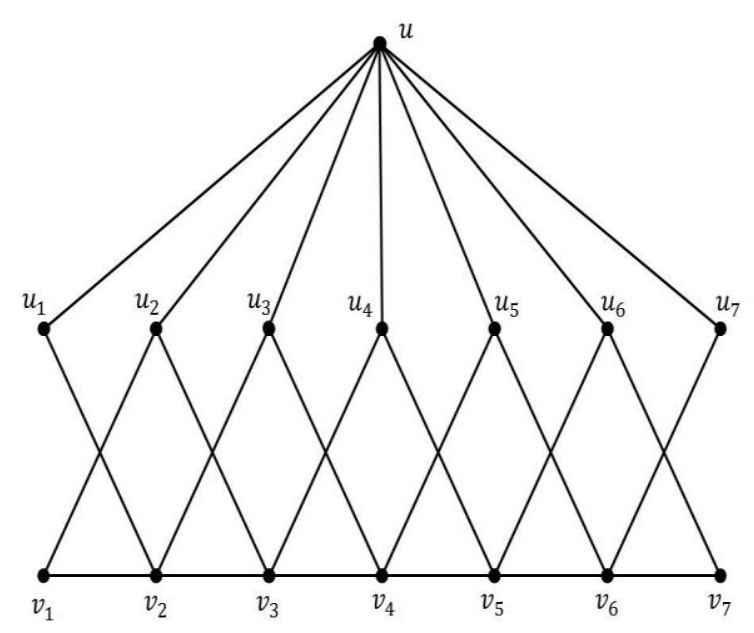

(a) Mycielskian graph of $\boldsymbol{P}_{7}$

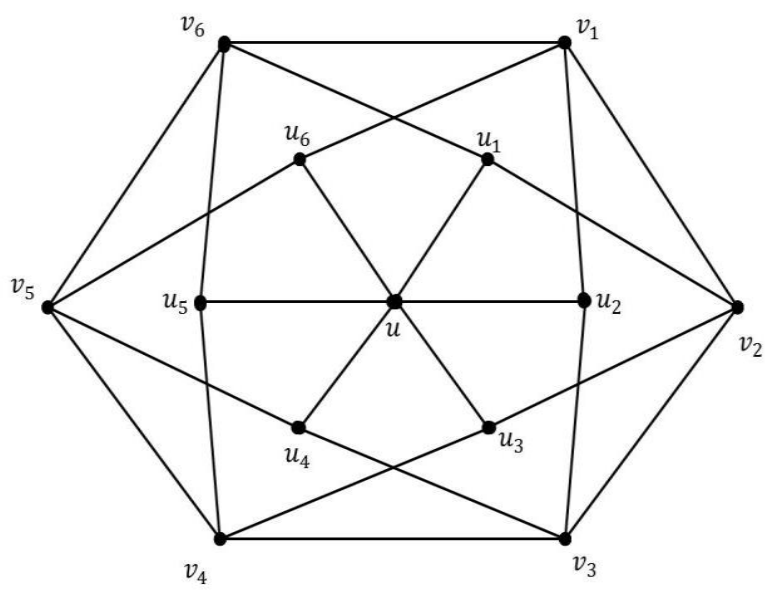

(b) Mycielskian graph of $\mathbf{C}_{6}$ Figure 1.1

\section{Theorem 3.2}

In a Cycle graph, for $n \geq 4, \gamma_{c}^{t c}\left[\mu\left(C_{n}\right)\right]=n-1$.

\section{Proof}

A cycle in a graph is a closed walk consists of a sequence ofvertices starting and ending at the same vertex, with each two consecutive vertices in the sequence adjacent to each other in the graph.

Let $V\left(C_{n}\right)=\left\{x_{i}: 1 \leq i \leq n\right\}$ be the set of vertices of $C_{n}$ taken in cyclic order. By the construction of Mycielski's graph,

$V\left(\mu\left(C_{n}\right)\right)=V\left(C_{n}\right) \cup\left\{y_{i}: 1 \leq i \leq n\right\} \cup$

$\{z\}$ and $E\left(\mu\left(C_{n}\right)\right)=E\left(C_{n}\right) \cup\left\{y_{i}(x): x \in N_{C_{n}}\left(x_{i}\right) \cup\right.$

$\{z\}, i=1,2, \ldots, n\}$. dominating set in $\left(C_{n}\right)$.

If $n=5$, fix the vertex set $\left\{u, u_{1}, u_{2}, u_{3}\right\}$ which is connected and its complement $V-D$ is the $\left\{v_{i} \cup u_{4}, u_{5}\right\}$ which is lie on a path. Therefore $\gamma_{c}^{t c}\left[\mu\left(C_{n}\right)\right]=n-1$.

Successively, hence $\gamma_{c}^{t c}\left[\mu\left(C_{n}\right)\right]=n-1$.

Result 3.3
Assume that $D=\left\{u, u_{i}: i=1,2, \ldots n-2\right\}$ is a blast

Let $\mathrm{G}$ be theMycielski's graph of Cycle graph such that $\mu\left(C_{n}\right)$ and $\overline{\mu\left(C_{n}\right)}$ have no isolated vertices and of order $2 n+1$.

(i) $\gamma_{c}^{t c}\left[\mu\left(C_{n}\right)\right]+\gamma_{c}^{t c}\left[\overline{\mu\left(C_{n}\right)}\right] \leq n+2$ and

(ii) $\gamma_{c}^{t c}\left[\mu\left(C_{n}\right)\right] \cdot \gamma_{c}^{t c}\left[\overline{\mu\left(C_{n}\right)}\right] \leq 3(n-1)$.

Proposition 3.4

For the graphs $\mu\left(C_{n}\right)$ and $\overline{\mu\left(C_{n}\right)}$ with maximum independence number $\beta_{0}\left(\mu\left(C_{n}\right)\right)$ and $\beta_{0}\left(\overline{\mu\left(C_{n}\right)}\right)$

$$
\text { (i) } \beta_{0}\left(\mu\left(C_{n}\right)\right)+\beta_{0}\left(\overline{\mu\left(C_{n}\right)}\right)=2 n
$$

(ii) $\beta_{0}\left(\mu\left(C_{n}\right)\right) \cdot \beta_{0}\left(\overline{\mu\left(C_{n}\right)}\right)=n^{2}$.

\section{Theorem 3.5}

In a Complete graph, wheren $\geq 3, \gamma_{c}^{t c}\left[\mu\left(K_{n}\right)\right]=3$.

\section{Proof}

A complete graph is a simple, connected,undirected graph in which every pair of distinct vertices is connected by a unique edge.

Let $V\left(K_{n}\right)=\left\{x_{i}: 1 \leq i \leq n\right\}$. By the construction of Mycielski's graph,

$V\left(\mu\left(K_{n}\right)\right)=V\left(K_{n}\right) \cup\left\{y_{i}: 1 \leq i \leq n\right\} \cup$

$\{z\} a n d E\left(\mu\left(K_{n}\right)\right)=E\left(K_{n}\right) \cup\left\{y_{i} x: x \in N_{K_{n}}\left(x_{i}\right) \cup\right.$

$\{z\}, i=1,2, \ldots n\}$.

Since $z$ is adjacent with each vertex of $\left\{y_{i}: 1 \leq i \leq\right.$ $n\}$, also $\mu\left(K_{n}\right)$ contains a n-clique.

Assume that $D=\left\{z, y_{i}, x_{i}: i=1,2, \ldots n\right\}$ is a blast dominating set in $\mu\left(K_{n}\right)$.

If $n=3$

Choose and fix any of the corresponding vertices $\left\{z, y_{1}, x_{2}\right\},\left\{z, y_{2}, x_{1}\right\},\left\{z, y_{3}, x_{3}\right\}$, which are connected and its complement $\left\{y_{2}, y_{3}, x_{1}, x_{3}\right\},\left\{y_{1}, y_{3}, x_{2}, x_{3}\right\},\left\{y_{1}, y_{2}, x_{1}, x_{2}\right\}$ forms a blast dominating set. Therefore, $\gamma_{c}^{t c}\left[\mu\left(K_{3}\right)\right]=$ 3.Successively, the assumption is true. Hence $\gamma_{c}^{t c}\left[\mu\left(K_{n}\right)\right]=$ 3.

\section{Result 3.6}

Let $\mathrm{G}$ be a Mycielski's graph of Complete graph such that $\mu\left(K_{n}\right)$ and $\overline{\mu\left(K_{n}\right)}$ have no isolated vertices of order $2 n+1$.

(i) $\gamma_{c}^{t c}\left[\mu\left(K_{n}\right)\right]+\gamma_{c}^{t c}\left[\overline{\mu\left(K_{n}\right)}\right]=6$ and

(ii) $\gamma_{c}^{t c}\left[\mu\left(K_{n}\right)\right] \cdot \gamma_{c}^{t c}\left[\overline{\mu\left(K_{n}\right)}\right]=9$.

Remark 3.7

For the graphs $\mu\left(K_{n}\right)$ and $\overline{\mu\left(K_{n}\right)}$ with the maximum independence number $\beta_{0}\left(\mu\left(K_{n}\right)\right)$ and $\beta_{0}\left(\overline{\mu\left(K_{n}\right)}\right)$,

$$
\text { (i) } \beta_{0}\left(\mu\left(K_{n}\right)\right)+\beta_{0}\left(\overline{\mu\left(K_{n}\right)}\right)=2 n
$$

(ii) $\beta_{0}\left(\mu\left(K_{n}\right)\right) \cdot \beta_{0}\left(\overline{\mu\left(K_{n}\right)}\right)=n^{2}$.

\section{Theorem 3.8}

In a Complete-bipartite graph $K_{m, n}$ where $m, n \geq$ $2, \gamma_{c}^{t c}\left[\mu\left(K_{m, n}\right)\right]=3$.

\section{Proof}

Let $V\left(\mu\left(K_{m, n}\right)\right)=\left\{x_{i}: 1 \leq i \leq m\right\}\left[\left\{y_{i}: 1 \leq j \leq\right.\right.$ $n\}$ and $E\left(K_{m, n}\right)=\cup_{i=1}^{m}\left[\left\{e_{i j}=x_{i} y_{j}: 1 \leq i \leq n\right\}\right.$. 
By Mycielski’s construction,

$V\left(\mu\left(K_{m, n}\right)\right)=V\left(K_{m, n}\right)\left[\left\{x_{i}^{\prime}: 1 \leq i \leq m\right\}\left[y_{j}^{\prime}: 1\right.\right.$ $\leq j \leq n[\{z\}]]$.

Choose the minimal blast dominating sets $D_{1}=$ $\left\{z, y_{1}, y_{m+n}\right\}$

$$
D_{2}=\left\{z, y_{2}, y_{m+n-1}\right\},
$$

$D_{n}=\left\{z, y_{i}, y_{m}\right\}$ and $\left|D_{1}\right|,\left|D_{2}\right| \ldots\left|D_{n}\right|=3 \quad$ which are connected and its complement $\langle V-D\rangle$ is triple connected. Hence $\gamma_{c}^{t c}\left[\mu\left(K_{m, n}\right)\right]=3$.

\section{Result 3.9}

Let $\mathrm{G}$ be a Mycielski's graph of Complete-bipartite graph $K_{m, n}$ such that $\mu\left(K_{m, n}\right)$ and $\overline{\mu\left(K_{m, n}\right)}$ have no isolated vertices of order $2 n+1$,

(i) $\gamma_{c}^{t c}\left[\mu\left(K_{m, n}\right)\right]+\gamma_{c}^{t c}\left[\overline{\mu\left(K_{m, n}\right)}\right]=6$ and

(ii) $\left.\gamma_{c}^{t c}\left[\mu\left(K_{m, n}\right)\right] \cdot \gamma_{c}^{t c} \overline{\left[\mu\left(K_{m, n}\right)\right.}\right]=9$.

\section{Proposition 3.10}

For the graphs $\mu\left(K_{m, n}\right)$ and $\overline{\mu\left(K_{m, n}\right)}$ with the maximum independence number $\beta_{0}\left(\mu\left(K_{m, n}\right)\right)$ and $\beta_{0}\left(\overline{\mu\left(K_{m, n}\right)}\right)$,

$$
\beta_{0}\left(\overline{\mu\left(K_{m, n}\right)}\right) \leq \beta_{0}\left(\mu\left(K_{m, n}\right)\right) .
$$

Theorem 3.11

In a Wheel graph, with $n \geq 3, \gamma_{c}^{t c}\left[\mu\left(W_{n}\right)\right]=3$.

\section{Proof}

A wheel in a graph is a graph formed by connecting a single vertex to all the vertices of a cycle. The wheel graph has $\mathrm{n}$ vertices and $2(n-1)$ edges. Let $V\left(W_{n}\right)=\left\{v \cup v_{i}\right.$ : $1 \leq i \leq n\}$ and $E\left(W_{n}\right)=\left\{v v_{i}: 1 \leq i \leq n\right\}$. By the Mycielski's construction, $V\left(\mu\left(W_{n}\right)\right)=V\left(W_{n}\right) \cup\left\{u u_{i}\right.$ : $1 \leq i \leq n\} \cup\{w\}$.In $\mu\left(W_{n}\right)$, each $u$ is adjacent with each vertex of $N_{W_{n}}(v)$, and $w$ is adjacent with each vertex of $\left\{u u_{i}: 1 \leq i \leq n\right\}$.

By the definition of Mycielskian, $v$ is adjacent with each of $\left\{v_{i}: 1 \leq i \leq n\right\} \cup\left\{u_{i}: 1 \leq i \leq n\right\}$. Assume that $D=\left\{w, u, v_{i}\right\}$ is a blast dominating set in $\mu\left(W_{n}\right)$.Suppose if $n=4$ then $V\left(\mu\left(W_{4}\right)\right)=V\left(W_{4}\right) \cup\left\{u u_{i}: 1 \leq i \leq 4\right\} \cup$ $\{w\}$.

Choose the minimal blast dominating sets $D_{1}=$ $\left\{w, u, v_{1}\right\}, D_{2}=\left\{w, u, v_{2}\right\}, D_{3}=\left\{w, u, v_{3}\right\}, D_{4}=\left\{w, u, v_{4}\right\}$ which are connected and their complements $V-D_{1}=$ $\left\{v \cup v_{2}, v_{3}, v_{4}\right\} \cup\left\{u_{i}: 1 \leq i \leq 4\right\}, V-D_{2}=\{v \cup$ $(v 1, v 3, v 4) \cup u i: 1 \leq i \leq 4, V-D 3=v \cup v 1, v 2, v 4 \cup u i: 1 \leq i \leq 4, V-$ $D_{4}=\left\{v \cup\left(v_{1}, v_{2}, v_{3}\right)\right\} \cup\left\{u_{i}: 1 \leq i \leq 4\right\} \quad$ are triple connected. Also $\left|D_{1}\right|,\left|D_{2}\right|,\left|D_{3}\right|,\left|D_{4}\right|=3$. Therefore, the assumption result holds. Hence $\gamma_{c}^{t c}\left[\mu\left(W_{n}\right)\right]=3$.

\section{Proposition 3.12}

Let $\mathrm{G}$ be the Mycielski's graph of Wheel graph such that $\mu\left(W_{n}\right)$ and $\overline{\mu\left(W_{n}\right)}$ have no isolated vertices and of order $2 n+1$,

(i) $\gamma_{c}^{t c}\left[\mu\left(W_{n}\right)\right]+\gamma_{c}^{t c}\left[\overline{\mu\left(W_{n}\right)}\right]=6$ and

(ii) $\gamma_{c}^{t c}\left[\mu\left(W_{n}\right)\right] \cdot \gamma_{c}^{t c}\left[\overline{\mu\left(W_{n}\right)}\right]=9$.

\section{Proof}

By the above theorem 3.11.the result is true.

\section{Result 3.13}

For the graphs $\mu\left(W_{n}\right)$ and $\overline{\mu\left(W_{n}\right)}$ with the maximum independence number $\beta_{0}\left(\mu\left(W_{n}\right)\right)$ and $\beta_{0}\left(\overline{\mu\left(W_{n}\right)}\right)$, $\beta_{0}\left(\overline{\mu\left(W_{n}\right)}\right) \leq \beta_{0}\left(\mu\left(W_{n}\right)\right)$

Theorem 3.14.

For the Tadpole graph $T_{m, n}$ where $n=1$ and $m \geq 4$, $\gamma_{c}^{t c}\left[\mu\left(T_{m, n}\right)\right]=n-1$.

\section{Proof}

Let $T_{m, 1}$ be the Tadpole graph with joining the cycle $C_{n}$ and path $P_{n}$ with $m=4, n=1$. Let $V\left(T_{m, 1}\right)=$ $\left\{V_{1}, V_{2}, \ldots V_{n}\right\}$. By the construction of Mycielski's graph,

$$
V\left(\mu\left(T_{m, n}\right)\right)=V\left(T_{m, 1}\right) \cup\left\{U_{i}: 1 \leq i \leq n\right\} \cup\{w\} \text { and }
$$

$E\left(\mu\left(T_{m, n}\right)\right)=E\left(T_{m, 1}\right) \cup\left\{U_{i} V: V \in N_{T_{m, n}}\left(V_{i}\right), i=1,2 \ldots n\right.$

Consider $\quad D=\left\{w \cup U_{i}: i=1,2 \ldots n-2\right\} \quad$ is the dominating set in $\mu\left(T_{m, n}\right)$ and $V-D=\left\{V_{i}: 1 \leq i \leq n-1\right\}$. Since $D$ is connected dominating set and whose complement is triple connected. Hence, $\gamma_{c}^{t c}\left[\mu\left(T_{m, n}\right)\right]=$ $n-1$.

\section{Proposition 3.15.}

For the graphs $\mu\left(T_{m, n}\right)$ and $\overline{\mu\left(T_{m, n}\right)}$ where $n=1$ and $m \geq 4$,

$$
\begin{gathered}
\text { (i) } \left.\gamma_{c}^{t c}\left[\mu\left(T_{m, n}\right)\right]+\gamma_{c}^{t c} \overline{\left[\mu\left(T_{m, n}\right)\right.}\right]=n+1 \\
\text { (i) } \gamma_{c}^{t c}\left[\mu\left(T_{m, n}\right)\right] \cdot \gamma_{c}^{t c} \overline{\left[\mu\left(T_{m, n}\right)\right]}=2(n-1) .
\end{gathered}
$$

Observation 3.16.

For the graphs $\mu\left(T_{m, n}\right)$ and $\overline{\mu\left(T_{m, n}\right)}, \quad \gamma_{\leq 2}\left(\mu\left(T_{m, n}\right)\right) \leq$ $\gamma\left(\overline{\mu\left(T_{m, n}\right)}\right) \leq \gamma_{c}^{t c} \overline{\left[\mu\left(T_{m, n}\right)\right.} \leq \gamma\left(\mu\left(T_{m, n}\right)\right) \leq \gamma_{c}^{t c}\left(\mu\left(T_{m, n}\right)\right)$

Theorem 3.17.

For the Fan graph $F_{1, n}$ where $n \geq 4$, then $\gamma_{c}^{t c}\left[\mu\left(F_{1, n}\right)\right]=$ 3.

Proof

The graph $F_{n+1}$ has $2 \mathrm{n}+3$ vertices. By the construction of Mycielski's graph,

$\left.V\left(\mu\left(F_{1, n}\right)\right)=V\left(F_{1, n}\right) \cup\left\{u \cup u_{i}: 1 \leq i \leq n\right)\right\} \cup\{w\}$ and $E\left(\mu\left(F_{1, n}\right)\right)=E\left(F_{1, n}\right) \cup\left\{u u_{i} v\right.$ : where $v \in N_{F_{n+1}}\left(v_{i}\right), 1 \leq i$ $\leq n)\}$

Choose $D=\left\{w \cup u_{i} \cup v: 1 \leq i \leq n\right\}$ as the dominating set of $\mu\left(F_{1, n}\right)$ and $V-D=\left\{v_{i}: 1 \leq i \leq n\right\}$. Here $D$ is a connected dominating set and its complement $V-D$ lies on the path. Therefore, $D$ forms a blast dominating set. Hence, $\gamma_{c}^{t c}\left[\mu\left(F_{1, n}\right)\right]=3$.

Result 3.18

For the graphs $\mu\left(F_{1, n}\right)$ and $\overline{\mu\left(F_{1, n}\right)}$ where $n \geq 3$,

$$
\begin{gathered}
\text { (i) } \left.\gamma\left[\mu\left(F_{1, n}\right)\right]+\gamma \overline{\left[\mu\left(F_{1, n}\right)\right.}\right]=4 \\
\text { (ii) } \left.\gamma\left[\mu\left(F_{1, n}\right)\right] \cdot \gamma \overline{\left[\mu\left(F_{1, n}\right)\right.}\right]=4 . \\
\text { (iii) } \left.\gamma_{c}^{t c}\left[\mu\left(F_{1, n}\right)\right]+\gamma_{c}^{t c} \overline{\left[\mu\left(F_{1, n}\right)\right.}\right]=6
\end{gathered}
$$




$$
\text { (iv) } \left.\gamma_{c}^{t c}\left[\mu\left(F_{1, n}\right)\right] \cdot \gamma_{c}^{t c} \overline{\left[\mu\left(F_{1, n}\right)\right.}\right]=9 .
$$

Theorem 3.19.

For the Snake graph $T_{n}$ where $n \geq 3, \gamma_{c}^{t c}\left[\mu\left(T_{n}\right)\right]=n$.

\section{Proof}

Let $\mathrm{G}$ be a triangular snake graph is a connected graph all of whose blocks are triangles. A triangularsnake graph is a triangular cactus whose block-cut vertex graph is a path. and it is obtained from the path $P=\left\{v_{1}, v_{2}, \ldots, v_{n+1}\right\}$ by joining $v_{i}$ and $v_{i+1}$ to a new vertex $u_{1}, u_{2}, \ldots, u_{n}$. By the construction of Mycielski's graph,

$$
V\left(\mu\left(T_{n}\right)\right)=V\left(T_{n}\right) \cup\left\{V_{i}: 1 \leq i \leq n\right\} \cup\left\{u_{i}: 1 \leq i \leq n+\right.
$$

$1\} \cup\{w\}$ and

$$
E\left(\mu\left(T_{n}\right)\right)=E\left(T_{n}\right) \cup\left\{V_{i} \cup u_{i} U V: N_{T_{n}}\left(U_{i} V_{i}\right), 1 \leq i \leq n\right\}
$$

Assume that $D=w \cup\left\{V_{i}: 1,2,3 \ldots n-1\right\}$ is a connected dominating set in $\mu\left(T_{n}\right)$ and $V-D=\left\{U_{i: 1} 1,2 . . n\right\} \cup$ $\left\{v_{i}: 1,2 \ldots n+1\right\}$ is triple connected in $\mu\left(T_{n}\right)$. Therefore $\gamma_{c}^{t c}\left[\mu\left(T_{n}\right)\right]=n$

\section{Result 3.20.}

For the graphs $\mu\left(T_{n}\right)$ and $\overline{\mu\left(T_{n}\right)}$ where $n \geq 3$,

$$
\begin{gathered}
\text { (i) } \left.\gamma\left[\mu\left(T_{n}\right)\right]+\gamma \overline{\left[\mu\left(T_{n}\right)\right.}\right] \leq n+2 \\
\text { (ii) } \gamma\left[\mu\left(T_{n}\right)\right] \cdot \gamma\left[\mu\left(T_{n}\right)\right] \leq(n+2)^{2} . \\
\text { (iii) } \gamma_{c}^{t c}\left[\mu\left(T_{n}\right)\right]+\gamma_{c}^{t c}\left[\mu\left(T_{n}\right)\right]=n+2 \\
\text { (iv) } \gamma_{c}^{t c}\left[\mu\left(T_{n}\right)\right] \cdot \gamma_{c}^{t c} \overline{\left[\mu\left(T_{n}\right)\right]}=2 n .
\end{gathered}
$$

\section{RELATIONSHIP WITH OTHER DOMINATION PARAMETERS}

In this section, some results and bounds related to distance-2 domination and independent distance-2 domination of mycielski's graphs are discussed. Also exact values of some special graphs areobtained.

Proposition 4.1.

Let $\mu\left(C_{n}\right)$ be a graph for $n \geq 3, \gamma_{\leq 2}\left(\mu\left(C_{n}\right)\right) \leq$ $i_{\leq 2}\left(\mu\left(C_{n}\right)\right)$.

Proof

Every independence distance- 2 dominating set of $\mu\left(C_{n}\right)$ is a distance-2 dominating set of $\mu\left(C_{n}\right)$. Thus, $\gamma_{\leq 2}\left(\mu\left(C_{n}\right)\right) \leq$ $i_{\leq 2}\left(\mu\left(C_{n}\right)\right)$.

\section{Proposition 4.2.}

For the graphs $G$ and $\bar{G}, n \geq 3, \gamma_{\leq 2}(G) \leq \gamma(\bar{G}) \leq \gamma(G) \leq$ $\gamma_{c}^{t c}(\bar{G}) \leq \gamma_{c}^{t c}(G)$ if $\mathrm{G}$ is any one of the graphs $\mu\left(C_{n}\right)$ or $\mu\left(K_{n}\right)$.

\section{Proposition 4.3}

For any graph $\mathrm{G}, \gamma_{c \leq 2}^{t c}(G)=\gamma_{\leq 2}(G)=1$ if and only if $\mathrm{G}$ is Mycielski's graph of $P_{n}$ or $C_{n}$ or $K_{n}$ or $K_{m, n}$ or $W_{n}$ or $F_{1, n}$ or $T_{m, n}$ or $T_{n}$.

\section{Proposition 4.4}

For theMycielski's graph of Complete-bipartite graphs $\mu\left(K_{m, n}\right)$ and $\overline{\mu\left(K_{m, n}\right)},(m, n \geq 2)$

(i) $\gamma\left(\mu\left(K_{m, n}\right)\right)=\gamma_{c}^{t c}\left(\mu\left(K_{m, n}\right)\right)$.

(ii) $\left.\gamma\left(\overline{\mu\left(K_{m, n}\right)}\right) \leq \gamma_{c}^{t c} \overline{\left(\mu\left(K_{m, n}\right)\right.}\right)$.

Proposition 4.5
For theMycielski's graph of Wheel graphs, $\mu\left(W_{n}\right)$ and $\left.\overline{\mu\left(W_{n}\right)} n \geq 3\right)$,

(i) $\gamma_{\leq 2}\left(\mu\left(W_{n}\right)\right) \leq \gamma\left(\mu\left(W_{n}\right)\right) \leq \gamma_{c}^{t c}\left(\mu\left(W_{n}\right)\right)$.

(ii) $\gamma_{\leq 2}\left(\overline{\mu\left(W_{n}\right)}\right) \leq \gamma\left(\overline{\mu\left(W_{n}\right)}\right) \leq \gamma_{c}^{t c}\left(\overline{\mu\left(W_{n}\right)}\right)$.

\section{Proposition 4.6}

For any graph $\mathrm{G}, \gamma_{\leq 2}(G) \leq i_{\leq 2}(G) \leq i(G) \leq \gamma(G) \leq$ $\beta_{0}(G)$ if and only if the graph $\mathrm{G}$ is any one of the following graphs $\quad \mu\left(C_{n}\right), \mu\left(P_{n}\right), \mu\left(K_{n}\right), \mu\left(K_{m, n}\right)$, $\mu\left(W_{n}\right), F_{1, n}, T_{m, n}$ and $T_{n}$.

\section{Proposition 4.7}

For any graph G, $\gamma_{c \leq 2}^{t c}(\bar{G})=\gamma_{\leq 2}(\bar{G})=1$ if and only if $\mathrm{G}$ is the Mycielski's graph of $C_{n}$ or $K_{n}$ or $K_{m, n}$ or $W_{n}$ or $F_{1, n}$ or $T_{m, n}$ or $T_{n}$.

\section{Observations 4.8}

(i) Every dominating set is a distance-2 dominating set if and only if $G$ is the Mycielski's graph of $P_{n}$ or $C_{n}$ or $K_{n}$ or $K_{m, n}$ or $W_{n}$ or $F_{1, n}$ or $T_{m, n}$ or $T_{n}$. But the converse is not true.

(ii) Every dominating set is a distance-2 dominating set if and only if $\bar{G}$ is the Mycielski's graph of $P_{n}$ or $C_{n}$ or $K_{n}$ or $K_{m, n}$ or $W_{n}$ or $F_{1, n}$ or $T_{m, n}$ or $T_{n}$.

(iii) Everydistance-2 dominating set is a blast dominating set if and only if $G$ is a Mycielski's graph of $P_{n}$ or $C_{n}$ or $K_{n}$ or $K_{m, n}$ or $W_{n}$ or or $F_{1, n}$ or $T_{m, n}$ or $T_{n}$.

(iv) Everydistance-2 dominating set is a blast dominating set if and only if $\bar{G}$ is a Mycielski's graph of $P_{n}$ or $C_{n}$ or $K_{n}$ or $K_{m, n}$ or $W_{n}$ or $F_{1, n}$ or $T_{m, n}$ or $T_{n}$.

4.9. Exact values and bounds of some standard graphs

(i) If any graph $\mathrm{G}=\mathrm{CP}_{\mathrm{k}}$ is the cocktail party graph of $\mathrm{k}$ vertices where $\mathrm{k}=2 \mathrm{n}$ for all $\geq 2$, then $\gamma_{c}^{t c}(G)=\gamma_{c \leq 2}^{t c}(G)=$ 1.

(ii) For the $\mathrm{n}$ - Andrasfai graph of $\mathrm{k}$ vertices where $\mathrm{k}=3 \mathrm{n}$ 1 for all $n \geq 1$,

$$
\gamma_{c}^{t c}\left(A_{k}\right)=\gamma_{c \leq 2}^{t c}\left(A_{k}\right)=1 .
$$

(iii) For any Harary graph $\mathrm{H}_{\mathrm{k}, \mathrm{n}}, \quad \gamma_{c}^{t c}\left(H_{k, n}\right)=$ $\gamma_{c \leq 2}^{t c}\left(H_{k, n}\right)=1=1$ for $k \geq 3$.

\section{BLAST DOMINATION NUMBER FOR THE MYCIELSKI'S GRAPH OF ZERO DIVISOR GRAPHS\& RESULTS}

This section deals with blast domination of Mycielski's graph of zero divisor graph and its complement graph and the bounds for these graphs are attained.The concept zero divisor graphs are introduced by I.Beck in 1988 and further studied by D.D.Anderson and M.Naseer

Example 5.1

$$
\Gamma\left(Z_{6}\right)=\{2,3,4\}
$$




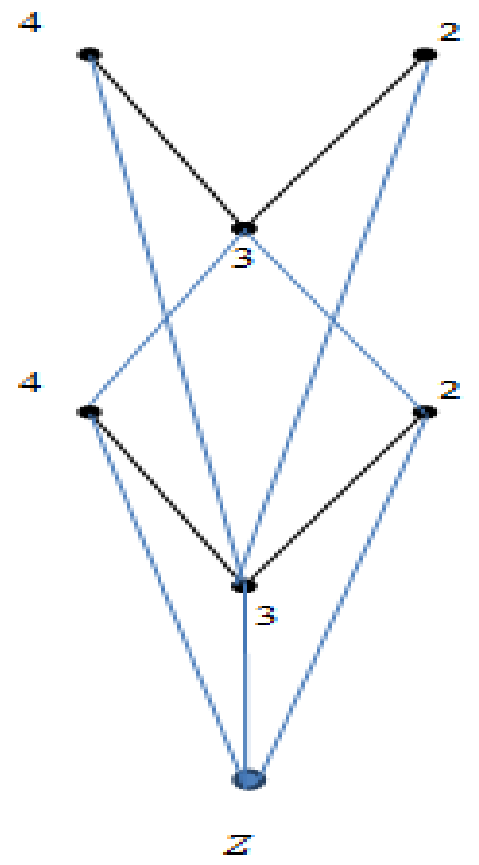

Figure 5.1TheMycielskian graph of $\Gamma\left(Z_{6}\right)$

\section{Proposition 5.2}

If $G=\mu\left(\Gamma\left(Z_{2 p}\right)\right)$ is the Mycielski's graph of star zerodivisor graph, then the blast domination number of $\mathrm{G}$ is given by, $\gamma_{c}^{t c}\left[\mu\left(\Gamma\left(Z_{2 p}\right)\right)\right]=3$.

\section{Result 5.3}

If the graphs $G=\mu\left(\Gamma\left(Z_{2 p}\right)\right)$ and $\bar{G}=\overline{\mu\left(\Gamma\left(Z_{2 p}\right)\right)}$ are the Mycielski's graphs of star zero divisor graph where $p$ is a prime number then

$$
\begin{gathered}
(i) \gamma\left[\mu\left(\Gamma\left(Z_{2 p}\right)\right)\right]+\gamma\left[\mu\left(\Gamma\left(Z_{2 p}\right)\right)\right]=4 \\
(i i) \gamma\left[\mu\left(\Gamma\left(Z_{2 p}\right)\right)\right] \cdot \gamma\left[\mu\left(\Gamma\left(Z_{2 p}\right)\right)\right]=4 \\
(i i i) \gamma_{c}^{t c}\left[\mu\left(\Gamma\left(Z_{2 p}\right)\right)\right]+\gamma_{c}^{t c}\left[\mu\left(\Gamma\left(Z_{2 p}\right)\right)\right]=5 \\
(i v) \gamma_{c}^{t c}\left[\mu\left(\Gamma\left(Z_{2 p}\right)\right)\right] \cdot \gamma_{c}^{t c}\left[\mu\left(\Gamma\left(Z_{2 p}\right)\right)\right]=6 .
\end{gathered}
$$

\section{Proposition 5.4}

For $G=\mu\left(\Gamma\left(Z_{n}\right)\right)$, if $n=3 p$ where $p$ is a prime number, the blast domination number of $\mathrm{G}$ is given by, $\gamma_{c}^{t c}\left[\mu\left(\Gamma\left(Z_{3 p}\right)\right)\right]=3$.

\section{Result 5.5}

If the graphs $G=\mu\left(\Gamma\left(Z_{3 p}\right)\right)$ and $\bar{G}=\overline{\mu\left(\Gamma\left(Z_{3 p}\right)\right)}$ are the Mycielski's of graphs zero divisor graph where $p$ is a prime number $(p>3)$ then

$$
\begin{gathered}
\text { (i) } \gamma\left[\mu\left(\Gamma\left(Z_{3 p}\right)\right)\right]+\gamma\left[\overline{\mu\left(\Gamma\left(Z_{3 p}\right)\right)}\right]=5 \\
\text { (ii) } \gamma\left[\mu\left(\Gamma\left(Z_{3 p}\right)\right)\right] \cdot \gamma\left[\mu\left(\Gamma\left(Z_{3 p}\right)\right)\right]=6 \\
\text { (iii) } \gamma_{c}^{t c}\left[\mu\left(\Gamma\left(Z_{3 p}\right)\right)\right]+\gamma_{c}^{t c}\left[\mu\left(\Gamma\left(Z_{3 p}\right)\right)\right]=6
\end{gathered}
$$

(iv) $\left.\gamma_{c}^{t c}\left[\mu\left(\Gamma\left(Z_{3 p}\right)\right)\right] \cdot \gamma_{c}^{t c} \overline{\mu\left(\Gamma\left(Z_{3 p}\right)\right)}\right]=9$.

Proposition 5.6

If $G=\mu\left(\Gamma\left(Z_{n}\right)\right)$ and $n=p^{2}$ where $p$ is an odd prime number,then the blast domination number of $\mathrm{G}$ is given by, $\gamma_{c}^{t c}\left[\mu\left(\Gamma\left(Z_{n}\right)\right)\right]=3$.

Proposition 5.7

For $G=\mu\left(\Gamma\left(Z_{n}\right)\right)$ and $n=p q$ where $=5, q=7$,then the blast domination number of $\mathrm{G}$ is given by, $\gamma_{c}^{t c}\left[\mu\left(\Gamma\left(Z_{n}\right)\right)\right]=3$.

Proposition 5.8

For the graphs $\mu\left(\Gamma\left(Z_{n}\right)\right)$ and $\mu\left(\Gamma\left(Z_{n}\right)\right)$,

$\gamma_{\leq 2}\left[\overline{\mu\left(\Gamma\left(Z_{n}\right)\right)}\right] \leq \gamma\left[\overline{\mu\left(\Gamma\left(Z_{n}\right)\right)}\right]$

$\leq \gamma\left[\mu\left(\Gamma\left(Z_{n}\right)\right)\right] \leq \gamma_{c}^{t c}\left[\mu\left(\Gamma\left(Z_{n}\right)\right)\right] \leq \gamma_{c}^{t c}\left[\overline{\mu\left(\Gamma\left(Z_{n}\right)\right)}\right]$

Observation 5.8

For the graphs $G=\mu\left(\Gamma\left(Z_{n}\right)\right)$ and $\bar{G}=\mu\left(\Gamma\left(Z_{n}\right)\right)$,

$$
\begin{aligned}
\gamma_{\leq 2}\left[\mu\left(\Gamma\left(Z_{n}\right)\right)\right] & =i_{\leq 2}\left[\mu\left(\Gamma\left(Z_{n}\right)\right)\right]=\gamma_{\leq 2}\left[\mu\left(\Gamma\left(Z_{n}\right)\right)\right] \\
& =i_{\leq 2}\left[\mu\left(\Gamma\left(Z_{n}\right)\right)\right]
\end{aligned}
$$

Proposition 5.9

If the graphs $G=\mu\left(\Gamma\left(Z_{n}\right)\right)$ and $\bar{G}=\overline{\mu\left(\Gamma\left(Z_{n}\right)\right)}$ are the Mycielski's graphs of the star zero divisor graph then (i) $\gamma_{\leq 2}\left[\mu\left(\Gamma\left(Z_{n}\right)\right)\right]+\gamma_{\leq 2}\left[\mu\left(\Gamma\left(Z_{n}\right)\right)\right]=2$

$$
\begin{aligned}
& (i i) \gamma_{\leq 2}\left[\mu\left(\Gamma\left(Z_{n}\right)\right)\right] \cdot \gamma_{\leq 2}\left[\mu\left(\Gamma\left(Z_{n}\right)\right)\right]=1 \\
& (\text { iii }) i_{\leq 2}\left[\mu\left(\Gamma\left(Z_{n}\right)\right)\right]+i_{\leq 2}\left[\mu\left(\Gamma\left(Z_{n}\right)\right)\right]=2 \\
& (\text { iv }) i_{\leq 2}\left[\mu\left(\Gamma\left(Z_{n}\right)\right)\right] \cdot i_{\leq 2}\left[\mu\left(\Gamma\left(Z_{n}\right)\right)\right]=1
\end{aligned}
$$

Proof

By the above observation 5.8 the result is true.

Obsevation 5.10

(i) Every distance-2 dominating set is a blast distance-2 dominating set in $\mu\left(\Gamma\left(Z_{n}\right)\right)$ and $\left[\mu\left(\Gamma\left(Z_{n}\right)\right)\right]$.Converse is also true.

(ii) Every independent distance-2 dominating set is a blast distance-2 dominating set in $\mu\left(\Gamma\left(Z_{n}\right)\right)$ and $\left[\mu\left(\Gamma\left(Z_{n}\right)\right)\right]$. Converse is also true.

Proof

The results follows from $\quad(i) \gamma_{\leq 2}\left[\mu\left(\Gamma\left(Z_{n}\right)\right)\right]$ $\leq \gamma_{c \leq 2}^{t c}\left[\mu\left(\Gamma\left(Z_{n}\right)\right)\right]$ and 
(ii) $i_{\leq 2}\left[\mu\left(\Gamma\left(Z_{n}\right)\right)\right] \leq \gamma_{c \leq 2}^{t c}\left[\mu\left(\Gamma\left(Z_{n}\right)\right)\right]$

\section{APPLICATIONS OF BLAST AND BLAST DISTANCE - 2 DOMINATING SETS}

We extended the concept of dominating sets to Blast and Blast distance - 2 dominating sets, there are more useful models to many real- world problems. Indeed, much of the motivation for the study of Blast and Blast distance -2 domination arises from problems involving locating optimally a hospital, police station, fire station, or any other emergency service facility.

\section{VII.CONCLUSION}

In this paper, we defined the notion of blast and blast distance-2 domination forMycielski's graph of $C_{n}, K_{n}, K_{m, n}$ and $W_{n}$ graphs. We attained many bounds on these two new parameters. Also Applications of Blast and Blast distance 2 dominating sets have been discussed. It would be interesting to determine the results for general graphs.

\section{REFERENCES}

1. BondyJ.A.and U.S.R. Murty, Graph theory, (Springer, Berlin, 2008).

2. ChartrandG and P. Zhang, Introduction to graph theory, (McGraw-Hill Inc., New York, 2005).

3. HaynesT.W.,HedetniemiS. $\mathrm{T}$ and SlaterP.J., Fundamentals of Domination in Graphs, Marcel Dekker, Inc.,New York, 1998.

4. Kulli.V.R.,(2012). Advances in domination theory, Vishwa International Publications, Gulbarga, India. Yorozu, M. Hirano, K.

5. Lin W, J. Wu, P.C.B. Lam and G. Gu, Several parameters of generalized Mycielskians,Discrete Appl. Math., 154(8)(2006), 1173\{1182\}.

6. G. Mahadevan, A. Ahila and S. Avadayappan,Blast domination number of a graph, Middle -East J. Sci. Res.,25(5) (2017), 977- 981International Journal of Pure and Applied Mathemati

7. Mahadevan .G,. Ahila . A and SelvamAvadayappan,Blast domination number for $\vartheta$ Obrazom, International Journal of Pure and Applied Mathematics, Volume 118 No. 7 2018, 111-117

8. Mahadevan .G,.Ahila . A and SelvamAvadayappan, Blast Domination Number on square and cube graphs,Contemp. Stud. Discrete Math., Vol. 1, No. 1, 2017, pp. 31-35

9. J.Paulraj Joseph, M.K.AngelJebitha, P.Chithra Devi and G.Sudhana, Triple connected graphs, Indian Journal of Mathematics and Mathematical Sciences, Vol.8, No.I(2012),61-75.

10. Tian.F and Xu J.M., A note on distance domination number of graphs, Australasian J. Combin.43(2009), 181-190. 